\title{
Competitive Judgments in a Business Simulation: A Comparison between American and Chinese Business Students
}

\author{
Eric G. Moore
}

University of Michigan, Ann Arbor

\begin{abstract}
A complex total business enterprise computer simulation was used as the setting for a study of judgments by Chinese and American business school students. The subjects were asked to make a series of decisions and give judgments about expected levels of competition for a new market opportunity in the simulation world. Decisions were compared across the groups based on the decision structure and content. The results confirm previous research as the American participants generated significantly more responses overall, and especially judgment-consistent responses than the Chinese participants. Analysis of the content of the decision representations found that the relative proportion of singular to distributional information in the responses was similar for both American and Chinese individuals when making decisions about their own teams, but the Chinese participants focused more heavily on distributional information when making judgments about the behavior of others. This implies that American and Chinese individuals focus on different aspectsof similar information and this may influence subsequent judgments. ㅇ 1998 John Wiley \& Sons, Inc.
\end{abstract}

The striking changes being experienced in the world of international 
business are important drivers of cross-cultural research. Dramatic political changes such as the collapse of the Soviet Union and the reunification of Germany, combined with economic changes, such as the rise of trading blocs such as the European Community, NAFTA, the Pacific Rim, and the Mercosur (Southern Common Market), have reshaped the world economy. In addition, corporations have increased their international exposure and there has been a tremendous increase in the use of multifunctional and multicultural teams. These political and economic forces have had significant impacts on international competition, and they must be recognized, assessed, and understood by scholars. Cross-cultural research is a crucial component in promoting this increased understanding.

This study extends the literature by addressing the need for better understanding about judgment differences involving competition by managers from different cultural backgrounds. The interaction between competitors is one of the most fundamental issues in business strategy. The dynamics of competition have been studied with the use of economic models such as game theory (Wilson, 1989) and empirically (Bowman \& Gatignon, 1995; Chen \& Miller, 1994; Gatignon, Anderson, \& Helsen, 1989; Robinson, 1988). Accuracy in perceiving these dynamics is a basic component of business strategy (Aaker, 1995; Porter, 1980). Consequently, the research question to be addressed in this study is whether differences occur between teams from the United States and Hong Kong for judgments of competitive intensity and, if differences exist, does the form of the decision representation created when making these competitive judgments differ as well? The research questions in this study are derived from the cross-cultural judgment literature and will be examined in an innovative simulation setting.

\section{Cultural Differences in Judgment}

What does the decision maker take into account in deliberating courses of action? A representation is the decision maker's personal characterization of the given situation (Yates, 1990). Such representations specify what is taken into account and what is ignored, and these factors can differ from person to person. Previous research has indicated that there are systematic differences in the kinds of things that Chinese and nonChinese include in their decision representations (Yates \& Lee, 1996). The study of decision making between Asian and Western cultures has often focused on the individual-level representation through an investigation of probabilistic thinking.

Probabilistic thinking has been defined as "the tendency to view the world in terms of uncertainty, the ascribing of different degrees of uncertainty to events, and the ability to meaningfully express that uncertainty either verbally or as a numerical probability" (Phillips \& Wright, 1977). In terms of methodology, prior research has tended to use prob- 
abilistic judgments as the primary metric in making cross-cultural comparisons. Probability judgments are a useful mechanism for gathering likelihood judgments because they permit explicit and careful trade-offs between the certainty of events and the seriousness of their consequences (cf. Raiffa, 1968; von Winterfeldt \& Edward, 1986; Winkler, 1972). Probability judgments are viewed as the result of an individual's feelings of uncertainty translated into a numerical response by internal decision processes (Phillips, 1970).

Cross-cultural research in probability judgments has generally used questionnaires to gather data, and two instruments have served as a basis for much of this research. These instruments are the View of Uncertainty Questionnaire (VUQ) and the Probability Assessment Questionnaire (PAQ). Both were developed by Phillips and Wright (1977) in their first study of cultural differences, and have been used in many of subsequent studies. Each of these instruments was designed to capture slightly different information. The VUQ is composed of general-knowledge questions and subjects were told to give a "reasonable response" to questions such as, "Will you catch a head cold in the next three months?" or "Is Baghdad the capital of Iraq?." Half of the questions were about events that had not yet happened, and others concerned factual matters that people may not be certain about. The PAQ includes questions such as, "Which is longer? (a) Panama Canal, (b) Suez Canal" and the subjects are asked to choose the right answer and also to indicate how sure they are of this answer by writing a percentage between 50 and 100. These approaches have been used to capture general information regarding both the correctness of the answers and the relative confidence of the subjects in their answers.

There are two main accuracy dimensions that are used to evaluate the answers given for probability judgments: calibration and discrimination. Probability judgments are well calibrated to the extent that the judgments attached to various events match the relative frequencies with which those events actually occur (Yates et al., 1989). For example, a subject would be perfectly calibrated if $60 \%$ of his or her probability assessments were correct when he or she indicated that they were $60 \%$ confident of the responses. Discrimination (or resolution) refers to the subject's tendency to say something different on those occasions when the target event is going to happen than on those when it is not (Yates, 1990). Probability judgments are discriminative or resolved to the extent that there is any contingency between those judgments and the occurrence or nonoccurrence of the target event. These accuracy dimensions have been applied to the VUQ and PAQ across several studies, and several interesting findings were derived.

The earliest studies of cultural differences in viewing uncertainty and assessing probabilities examined British and Asian subjects' responses to uncertain situations (Phillips \& Wright, 1977; Wright \& Phillips, 1980). From their empirical studies, Phillips and Wright consistently 
found that Asian judgments were more positively biased than British subject's assessments for almanac-based questions. Positive bias is related to the individual's confidence in his or her judgment. This means that the person's average judgment that he or she selected the right answers to various questions outstrips the objective rate at which the person really does pick correct answers (Yates, 1990). This finding was generalized across managerial and clerical workers, as well as student populations (Phillips \& Wright, 1977; Wright et al., 1978; Yates et al., 1989).

An explanation for these strong positive biases may have to do with the relative ease in spontaneous generation of alternatives to the correct answer. One systematic difference is the relative amount of positive and negative information used in a decision representation. Yates, Lee, and Shinotsuka (1992) found that Chinese subjects generated fewer reasons against the correctness of the answers they selected than Japanese or American subjects. Even though the subjects tried very hard, they produced far fewer reasons disagreeing with the answers they ultimately chose than their Japanese and American counterparts, and also fewer reasons overall.

\section{The Use of Information in Judgments}

A second area of interest between American and Asian individual judgments involves the type of information used in making the predictive judgments. Kahneman and Tversky (1979) were among the first researchers to study the importance of the type of information used in making judgments in their investigation of the planning fallacy. The planning fallacy is defined as the tendency to hold a confident belief that one's own project will proceed as planned, even while knowing that the vast majority of similar projects have run late (Kahneman \& Tversky, 1979). Kahneman and Tversky (1979) found that people commonly use two forms of information when predicting task completion. For planning tasks, singular information relates to aspects of the specific target task that might lead to longer or shorter completion times. Distributional information concerns how long it took to complete other, similar tasks (Beuhler, Griffin, \& Ross, 1994). These two types of information can be generalized to other decision-making situations.

Kahneman and Tversky (1979) suggested that people who focus on case-based or singular information adopt an internal perspective. They will use the most current information as the basis for their judgments. In contrast, people who primarily consider distributional information have an external perspective and will look at past experiences that are similar to the current situation. The two general approaches to judgments or predictions differ primarily in whether individuals treat the target task as a unique case or as a composite of similar experiences.

People commonly use both forms of information when making a judg- 
ment. However, there are contexts where people use predominately one form of information in the service of their predictions. From the research on planning, people typically adopt an internal perspective and use singular information when predicting their own completion times; they seemingly fail to consider such relevant distributional information as their previous experiences with similar tasks (Kahneman \& Tversky, 1979). Research on the use of background data such as base rates suggests that Americans tend to neglect background data when they possess case-based information on which to form their judgments (BarHillel, 1980; Kahneman \& Tversky, 1982). However, people may adopt an external perspective when predicting the activity of others. Congruent with this external perspective, people will increasingly focus on distributional information when they make predictions about competitive activities of others (Kahneman \& Tversky, 1982). This distributional information could be based on their own past experiences in business (personal base rates) or the experiences of others (population base rates).

In contrast to the American pattern of use of singular and distributional information, the Chinese teams may have a different style of information use. In a macro-level comparison with American culture, the Chinese culture places a relatively higher premium on past activities. Things that are valued in Chinese society are facts and procedures that have been accepted as having proved their worth through the empirical test of time (Yates \& Lee, 1996). Given Chinese assumptions, the most reasonable path to knowledge would be activities intended to achieve an understanding of what is already known and accepted as correct. Consequently, the Chinese participants may be expected to use relatively more distributional information in judgments of competitive activity for both the team's own decision and for the perceptions of the activity of other teams.

\section{Cross-Cultural Research Methods}

Traditional methods for cross-cultural research, such as case studies, survey research, and laboratory experiments, all have advantages and disadvantages. Essentially, case studies and ethnographic research are helpful in identifying possible ideas for theory development, but are difficult and time-consuming to conduct, and their generalizeabilty is often limited. Surveys allow for descriptive statistical comparison of cross-cultural data. Unfortunately, construct validity is often suspect due to the difficulty of assuring cross-cultural equivalence of constructs and response sets. Laboratory experiments allow theory testing under conditions of high experimental control, but criticism of these statistically powerful designs centers on the possibility that the experimental stimulus is often a poor substitute for the real-life variables in question. Cross-cultural research would ideally benefit from laboratory research 
designs, but few experimental stimuli possess the power necessary to engage participants for long periods of time or allow sufficient behavior to test behavioral theories. Simulation games such as complex business simulations may provide ideal vehicles for the conduct of theory testing, and especially for cross-cultural research.

\section{The Use of Business Simulations}

Computer simulations have been used in business education for over 30 years, primarily to teach fundamental skills and concepts to students. Simulations have gained widespread acceptance in business schools, as evidenced by a survey by Faria (1987), which found that $95 \%$ of all 4-year American Assembly of Collegiate Schools of Business were using business simulations in at least one course. A simple simulation may focus on only a few decisions by an individual decision maker. The most sophisticated simulations model marketing processes such as market segmentation and brand equity and they give the decision maker control over marketing-mix decisions of product design, price, distribution, advertising, and promotion mix in a highly competitive setting. An example of such a sophisticated simulation is the BRANDMAPS computer simulation.

BRANDMAPS (Chapman, 1988) simulates an economy consisting of several markets (labeled as countries) that are served by the competing firms. Each of these firms has financial, production, and market histories. The mechanism of the simulation is a series of decision periods, each reflecting one quarter of the simulation year. Each decision period can be broken down into a complex process of acquiring information, evaluating, and making choices to direct the firm through the simulation environment.

The decision process involves a series of input screens where the team members systematically make decisions with respect to each product line, and overall sales force and production issues. Each firm has a set of products that are formulated from a combination of input materials. The various combinations of materials influence the way the products are preferred within the market. Market research studies can be purchased, including conjoint and preference tests to determine how close the product formulations match market ideal points. The team must decide on unit price for each market, advertising spending, advertising message, promotional spending, type of promotion, and a dealer rebate.

As a program, BRANDMAPS is one of the richest in complexity. The program has a series of checks to detect impossible values and particularly extreme or poor decisions. Additional features include a static spreadsheet-type analysis support system to model the effects of changing parameters. The complexity of the simulation structure and the high 
level of involvement by the players makes BRANDMAPS a very good environment for researching business issues.

Simulations are an attractive setting for research because they have a natural learning objective for the participants (Fletcher, 1971). Simulations also provide for opportunities for randomization of participants for experimental and control groups to increase external validation of the results. The decision process of the participants embodies the perceptions and assumptions of the group members, and these perceptions and assumptions can be examined in the dynamic setting of a simulation. The decision-making process is often implicit and the structured nature of an experiment helps to make the perceptions explicit. This allows the researcher to identify differences that may exist between and within the groups in the study under more realistic conditions than simple questionnaires and experiments.

\section{Summary}

There are two main research questions derived from the cross-cultural literature to be tested with the use of a business simulation. The first question is to examine if previous findings, such as Yates et al. (1992), from questionnaire-based laboratory settings extend to the more naturalistic decision-making environment of the business simulation. The second research question is to test whether American and Chinese have different patterns of information usage with respect to the relative proportions of singular and distributive information for both their own decisions and judgments of others.

\section{METHOD}

\section{Participants}

The pool of participants consisted of 41 Master's-level students at the School of Business at the University of Michigan, who participated in the study as an exercise in an advanced marketing strategy course in the fall of 1994. The 21 Chinese students were part of a special M.B.A. program with Cathay Pacific Airlines. The special nature of this program limited the available sample size. As the program was not repeated, the sample size could not be enlarged. The 20 American students were first- and second-year M.B.A. students and were similar with respect to industry and game experience. All of the written materials were provided in English, but the groups were allowed to use any language they desired to discuss the relevant issues. The Chinese groups primarily spoke in Cantonese, and both groups completed the worksheets in English. 


\section{Materials}

The Chinese students formed one simulation world of five teams and the American students were in an independent simulation world, also with five teams. The simulation served only as the setting for the experimental task, and the teams did not have to interact with the computer during the task. The BRANDMAPS simulation initially consisted of four markets (France, Germany, U.S., and the U.K.), in which to sell the products designed by the teams.

The experimental design involved presenting each team with information about the opportunity to sell products in a new fifth market (Japan). Each team was given the same standard market research reports generated in the BRANDMAPS simulation that detailed the size of the market, potential growth rates, and data on feature and price preferences of the customers to understand the factors involved with creating a customized product for the Japanese market. These research reports were designed to make the market fairly attractive and served as the singular information provided to each team. The students had played BRANDMAPS for six decision cycles before the experiment was conducted, and they were comfortable with the game mechanics and research reports. The subjects were directed before the experiment to bring their historical records of the past competitive activity in the game world to ensure the availability of this information for use in making their judgments. These historical reports served as the source of distributional information for the experiment.

The new market opportunity was designed to allow the teams to enter the new market despite any existing problems or successes by a team. This was an important feature of the experimental design to reduce the effect of possible past failures and successes as primary explanatory reasons for making decisions.

\section{Design and Procedure}

The simulation allowed the researcher to manipulate key variables designed to elicit actual decision-making behavior. Hypotheses testing could then occur. These controlled manipulations are identified as three tasks for the research subjects.

Task 1: Evaluation of the Attractiveness of the Japanese Market. Each team was asked to evaluate the attractiveness of Japanese market opportunity for the computer simulation environment based on the information provided. Each team indicated their perception of the attractiveness of the new market opportunity by placing a mark on a semantic-differential scale with 1 meaning, "Not Very Attractive" and 5 meaning, "Very Attractive." The teams were then instructed to list the 
criteria they used in making this judgment and to rate the importance of each criterion.

\section{Task 2: Decision of Own Team Entry for the Japanese Market.} The first assignment for each participant was to make a decision of whether their team should enter the Japanese market in the next decision period. Each person was then instructed to write down the criteria they used in making this decision, including criteria that could be different from those used in evaluating the attractiveness of the market.

\section{Task 3: Individual Judgments of Competing Team Entry for the} Japanese Market. Subjects were asked individually to make a judgment of whether each of the other four teams would enter the Japanese market in the next decision period and to give an explanation for why they would do so. Each subject indicated on a worksheet the probability the team would enter (from 0, meaning no chance, to $100 \%$, meaning perfect certainty). In addition, each subject made a composite judgment for all teams with the supporting criteria used in making these judgments. The individual assessments were then collected by the researcher and the team was directed to make a composite judgment of the entry probability for each competitor. Specifically, the subject was told, "Based on your experiences, what is the probability that each team will enter the market?", and to "Explain your key reasons influencing your judgment."

\section{Coding}

A data set was created from the worksheet responses. Two coders who were naive to the purposes of the study were trained and coded responses independently. The responses from Task 2 on the evaluation of their own team decision and the judgment of the other teams from Task 3 were coded with respect to positive, neutral, or negative valence, and by the type of information as singular or distributional. Initial agreement between the two coders was $88 \%$ for the valence of a response and $84 \%$ for the type of information. Differences were settled by discussion and mutual agreement.

The valence of a response was evaluated in terms of how favorable the individual appraised the item in the direction of the Japanese market entry decision. An example of a positive item would be, "The large market size makes the Japanese market a potentially attractive market." An example of a neutral item would be, "The cost to produce a product for the Japanese market is about the same as for the German market", and a negative item would be, "The Japanese market appears to be price sensitive, which could negatively impact the ability to raise prices in the future." 
For the coding of the responses with respect to the type of information, an example of a singular comment given by a team member would be, "The other team's reasons for entering the Japanese market will be size and growth in population and income (basically the same reasoning as we did in evaluating the market)", and an example of a distributional response would start, "By the experience in other regions . . ..".

Task 1 served as a manipulation check of the validity of the task. The question was designed to determine if the Chinese and American teams perceived the attractiveness of the Japanese market opportunity in comparable ways. The evaluations were very similar with a mean of 2.65 for the American teams and a mean of 2.56 for the Chinese teams, on the 5-point attractiveness scale. This result indicates that both of the groups gave similar evaluations of the market opportunity and that there were no significant differences in the basic perceptions of the situation.

\section{RESULTS}

The data were analyzed with respect to each Chinese and American own-team decision of whether to enter the market opportunity from Task 2 and then for the other-team evaluation from Task 3 . Analyses were conducted to test for the statistical reliability of the differences between the American and Chinese groups with respect to the total number of responses volunteered by the participants, the relative proportion of judgment-consistent criteria they offered, and the relative proportions of singular and distributional criteria the subjects used in making their decisions. The limited sample size and the difficulty in replication with a second experiment makes any interpretation exploratory rather than conclusive. Because of the small sample sizes, these analyses were conducted first with the use of standard statistical tests and then also tested with nonparametric statistics to confirm significant findings. The proportional data were arcsine transformed to "stretch the tails" (Cohen \& Cohen, 1983) in order to achieve a scale of measurement that was more nearly linearly related to other variables.

\section{Own-Team Decision Structure}

The first step of the methodology was to compare the average total number of criteria proposed by the two groups. The research by Yates et al. (1992) suggests that Chinese teams would be expected to generate fewer reasons overall than the American teams. The analysis for equality of means confirms this finding $(t(39)=4.80, p=.00$; Wilcoxon rank sum $W=565, Z=-3.99, p=.00, n=41$ ). The tabulation of frequencies shows that the Americans generated approximately twice as many responses (53 to 32). 
The second step also follows from the work of Yates et al. (1992). In their study, Chinese individuals generated fewer reasons against the correctness of the answers the subjects selected than did the Americans. For this experiment, this would imply that Chinese teams would generate fewer positive criteria when they chose not to enter the Japanese market than American teams, and also fewer negative criteria for the teams that chose to enter the new market.

For both the American and Chinese teams, the responses generally supported the direction of the decision. The tabulation of frequencies shows that the Americans generated slightly lower judgment-consistent responses ( $38 \%$ to $42 \%$ ), but three times as many judgment-inconsistent responses than the Chinese (18\% to 6\%). The statistical analysis confirms the lack of a significant difference for the level of consistent judgments $(t(39)=-0.26, p=.79)$ and a significant difference in the judgment inconsistence in the responses between the groups $(t(39)=2.52$, $p=.02$; Wilcoxon rank sum $W=491, Z=-1.98, p=.05, n=41$ ).

\section{Own-Team Decision Content}

The next step was to examine the relative proportions of singular and distributional responses between the two groups. For the own-team judgment of entry from Task 2 , both the American and Chinese groups were expected to use more singular than distributional information. In addition, the Chinese were expected to have higher levels of distributional information than the Americans. The raw frequencies show that both groups used an almost identical, and relatively high, proportion of singular information (74\% American, 76\% Chinese) in their written responses. Statistical tests indicated that there were no significant differences between the two groups in the levels of information for the own judgment $(t(39)=.69, p=.29)$. The finding was consistent with theory about judgments of own activities by North American subjects

(Kahneman \& Tversky, 1979). The result that the overall levels of singular and distributional information were very similar between the Chinese and American individuals was very interesting and indicates that both groups approached the entry question for their own team with similar patterns of information use.

\section{Other-Team Judgment Structure}

As with the own-team judgment, the first step of the methodology was to compare the average total number of criteria between the two groups. The expectation for the other-team judgment remains the same as the own team in that the Chinese teams would be expected to generate fewer reasons overall than the American teams. The analysis confirms this finding $(t(39)=3.41, p=.00$; Wilcoxon rank sum $W=619$, $Z=-5.29, p=.00, n=41)$. The tabulation of frequencies show that 
consistent with the own-team responses, the Americans generated twice as many responses (98 to 49 ) as the Chinese.

The analysis of the consistency of the responses also followed a similar pattern to the own-team responses. The tabulation of raw frequencies showed that the Americans generated similar levels of judgmentconsistent responses $(44 \%-42 \%)$, and also higher levels of judgmentinconsistent responses than the Chinese $(20 \%-12 \%)$. The statistical analysis confirms the lack of a significant difference in the judgmentconsistent level $(t(39)=-1.54, p=.13)$ and approaching a significant difference for the level of judgment inconsistence in the responses between the groups $(t(39)=-1.74, p=.09$; Wilcoxon rank sum $W=470, Z=-1.66, p=.10, n=41)$.

\section{Other-Team Judgment Content}

In contrast to the entry decision for their own team, the judgments of whether the other teams would choose to enter the Japanese market in Task 3 were expected to involve more distributive information for both the American and Chinese individuals. The Chinese were still expected to have an overall higher proportion of distributional information due to cultural considerations. The frequencies show that the Americans used $76 \%$ singular information and the Chinese used only $55 \%$ singular for their judgments. The test for differences in the level of information were significant $(t(39)=3.45, p=.00 ; W=346, Z=-2.11, p=.03$, $n=41)$.

A useful comparison is to examine the relative proportions across the own-decision and other-judgment for both the American and Chinese groups. The results indicate that the relative proportion of information use for the Americans was fairly similar for both the own-decision and the other-judgment $(t(18)=.66, p=.26)$. However, the Chinese pattern does show a larger percentage of distributional information than the pattern from their own-team entry judgment (45\% other vs. $24 \%$ own) and statistical tests confirm that this difference is significant $(t(19)=$ $1.69, p=.02$; Wilcoxon matched-pairs signed-ranks test $Z=-2.16$, $p=.03, n=21$ ).

\section{DISCUSSION}

In a business simulation setting, a series of tasks were conducted to explore possible differences in the perception of competitive activity between Chinese and American teams. The results indicate that there are differences in the level of responses created while making these decisions, as well as the type of information used in the decision representation.

The results of both the number and pattern of criteria support the 
previous research findings that Chinese individuals are expected to generate fewer reasons than American individuals, and particularly fewer against their judgments (Yates et al., 1992). The Chinese teams not only generated fewer reasons overall, but interestingly they also did not generate any positive criteria for the decision not to enter condition in either their own-team decision or for the other-team judgment. This finding indicates that more focused research on this aspect should be conducted, as it may be more difficult for the Chinese participants to create judgment-inconsistent criteria in this particular direction.

The examination of the relative levels of singular and distributional information use revealed that the Americans consistently held an internal perspective and focused primarily on singular information during the decision process. In contrast, the Chinese pattern of responses indicates the use of an internal perspective when making their own-team decision, and an external perspective when making the other-team judgment. These differences in patterns imply that for decisions that involve cross-cultural teams, the two groups may place different emphasis on information in their judgments of others.

Although the Chinese subjects were hypothesized to use a greater proportion of distributional information in making all of their decisions, the similar level of information use to the American individuals by the Chinese for the own-entry task may be explained by task characteristics. The high level of singular information may have been an unintended consequence from the sequence of tasks in the experiment. All of the participants were given the stimulus materials about the new Japanese market, and these materials were the only sources of data for making their evaluation of the attractiveness of the brand-new market. Consequently, when they were asked to give their judgment about their own team's decision to enter the Japanese market, the salient features (singular information) from the stimuli may have been more strongly activated (e.g., through the use an availability heuristic) than other items generated from distributional information. This possible salience effect should not be overemphasized, because the subjects did include a fair amount (around 25\%) of distributional information in their written explanations of their own decisions, but the use of heuristics in crosscultural decision making needs to be investigated further.

The overall high level of singular information used in the decision representations is not very surprising. The future nature of prediction may prevent individuals from looking back at previous experiences. People may attend to their past experiences but nevertheless fail to incorporate this information into their predictions (Beuhler et al., 1994). Often it may be difficult to detect the appropriate set of past experiences; the various instances seem so different from each other that individuals cannot compare them meaningfully (Kahneman \& Tversky, 1979). However, the setting of a simulated business world mitigates this difficulty by providing all of the participants with a shared set of experiences 
through the previous game cycles. The value of the simulated setting cannot be overstated, as it would be almost impossible to conduct a similar study in a competitive environment.

A limitation in the programming of the computer simulation design (a maximum of nine teams) and time constraints on the availability of the limited number of Chinese students prevented running all 10 teams within the same world. A shared world would have been a more preferred situation for conducting the experiment and would have allowed for more direct comparison of the shared decision variable context rather than comparing two similar, yet separate, worlds. However, both of these independent simulation worlds were started with the same underlying parameter values to maintain consistency between the groups, and the game experience should have been very similar for all of the participants.

Implications for marketing are grounded in the finding that Chinese and American decision-making profiles are different. Although this research focused on market entry behavior, some implications for other aspects of marketing may be drawn. The Chinese use of distributional data implies that there may be a tendency to weight such information greater than immediate situational information. In consumer behavior situations, this tendency may have impacts on such constructs as customer satisfaction, constructs which are functions of cumulative experiences with a product. Although intriguing, these statements were not directly related to the purpose of the study. This study explored the fundamental issue of whether or not cross-cultural differences in decision making did exist and to preliminarily identify some theory-based reasons for differences. Use of simulations in experimental modes allowed this study to tease out some differences. Future research should be conducted with larger samples, to enable more rigorous testing.

As a final note, the U.S. and Hong Kong groups were surveyed after completing the BRANDMAPS simulation. Participants from both groups stated that they felt that they were highly engaged by the simulation and felt that simulation situations were realistic and challenging intellectually. Participants unanimously felt that they had learned a great deal about the development of marketing strategy and that their involvement in the business simulation was a worthwhile experience. Consequently, computer simulations may be used increasingly as tools to teach marketing concepts and as vehicles for decision-making research.

\section{REFERENCES}

Aaker, D. (1995). Strategic market management. New York: John Wiley \& Sons. Bar-Hillel, M. (1980). The base-rate fallacy in probability judgments. Acta Psychologica, 44, 211-233. 
Beuhler, R., Griffin, D., \& Ross, M. (1994). Exploring the "planning fallacy": Why people underestimate their task completion times. Journal of Personality and Social Psychology, 67, 366-381.

Bowman, D., \& Gatignon, H. (1995). Determinants of competitor response time to a new product introduction. Journal of Marketing Research, 32, 42-53.

Chapman, R. (1988), BRANDMAPS: The competitive marketing strategy game. Englewood Cliffs, NJ: Prentice-Hall.

Chen, M., \& Miller, D. (1994). Competitive attack, retaliation and performance: An expectancy valence framework. Strategic Management Journal, 15, 85-102.

Cohen, J. \& Cohen, P. (1983). Applied multiple regression / correlation analysis for the behavioral sciences. Hillsdale, NJ: Lawrence Erlbaum Associates.

Faria, A. (1987). A survey of the use of business games in academia and business. Simulations and Games, 18, 207-224.

Fletcher, J. L. (1971). Evaluation of learning in two social studies simulation games. Simulation and Games, 2, 425-454.

Gatignon, H., Anderson, E., \& Helsen, K. (1989). Competitive reactions to market entry: Explaining interfirm differences. Journal of Marketing Research, $26,44-55$.

Kahneman, D., \& Tversky, A. (1979). Intuitive prediction: Biases and correction procedures. TIMS studies in management science, 12, 313-327.

Kahneman, D., \& Tversky, A. (1982). Judgment under uncertainty: Heuristics and biases. Cambridge, England: Cambridge University Press.

Phillips, L. D. (1970). The true probability problem. Act Psychologica, 34, 254-264.

Phillips, L. D., \& Wright, G. N. (1977). Cultural differences in viewing uncertainty and assessing probabilities. In H. Jungermann \& G. de Zeeuw (Eds.), Decision making and change in human affairs (pp. 507-519). Dordrecht, Holland: Reidel.

Porter, M. (1980). Competitive strategy. New York: Free Press.

Raiffa, H. (1968). Decision analysis. Reading MA: Addison-Wesley.

Robinson, W. (1988). Marketing mix reactions to entry. Marketing Science, 7, $368-385$.

von Winterfeldt, D., \& Edward, W. (1986). Decision analysis and behavioral research. New York: Cambridge University Press.

Wilson, R. (1989). Deterrence in oligopolistic competition. In P. C. Stern, R. Axelrod, R. Jervis \& R. Radner (Eds.), Perspectives on deterrence (pp. 157190). New York: Oxford University Press.

Winkler, R. L. (1972). Introduction to Bayesian inference and decision. New York: Holt, Rinehart \& Winston.

Wright, G. N., \& Phillips, L. D. (1980). Cultural variation in probabilistic theory: Alternative ways of dealing with uncertainty. International Journal of Psychology, 15, 239-257.

Wright, G. N., Phillips, L. D., Whalley, P. C., Choo, G. T., Ng, K. O., Tan, I., \& Wisudha, A. (1978). Cultural differences in probabilistic thinking. Journal of Cross-Cultural Psychology, 9, 285-299.

Yates, J. F. (1990). Judgment and decision making. Englewood Cliffs, NJ: Prentice-Hall. 
Yates, J. F., \& Lee, J. -W. (1996). Chinese decision making. In M. H. Bond (Ed.), Handbook of Chinese psychology. Hong Kong: Oxford University Press.

Yates, J. F., Lee, J.-W., \& Shinotsuka, H. (1992). Cross-national variation in probability judgment. Paper presented at the Annual Meeting of the Psychonomic Society, St. Louis.

Yates, J. F., Zhu, Y., Ronis, D. L., Wang, D. F., Shinotsuka, H., \& Toda, M. (1989). Probability judgment accuracy: China, Japan and the United States. Organizational Behavior and Human Decision Processes, 43, 145-171.

Correspondence regarding this article should be sent to: Eric G. Moore, Department of Marketing, School of Business, University of Michigan, 701 Tappan St., Ann Arbor, MI 48109 (egmoore@umich.edu). 